\title{
A case of primary actinomycosis and secondary eumycetoma in anterior abdomen wall - A case report
}

\author{
Agil. S. ${ }^{1}$, Ratna $^{2}$, N. Bharath ${ }^{3}$, A. Rekha ${ }^{4}$ \\ ${ }^{1}$ Dr. Agil. S, Post Graduate, ${ }^{2}$ Dr. Ratna, Post Graduate, ${ }^{3}$ Dr. N. Bharath, Assistant Surgeon, ${ }^{4}$ Dr. A. Rekha, Professor, all \\ authors are affiliated with Saveetha University, No.162, Poonamalle High Rd, Velappanchavadi, Chennai, Tamil Nadu, \\ India.
}

Corresponding Author: Dr. Agil. S, Post Graduate, Saveetha University, No.162, Poonamalle High Rd, Velappanchavadi, Chennai, Tamil Nadu, India. Email: agil_amirthalingam@yahoo.co.in

\begin{abstract}
Actinomycosis of abdomen accounts to only 10-20\%. We report a 58-year-old diabetic man who presented with abdomen pain and swelling for 10 days duration associated with fever. Abdominal computed tomography showed a large thick walled peripheral enhancing collection between lateral abdominal wall and capsular surface of right lobe of liver with extension into right lateral abdominal wall muscle at level $12^{\text {th }}$ rib. Laparotomy and peritoneal wash was performed. Intra-operative pus pocket $300 \mathrm{ml}$ drained from 10, 11 and $12^{\text {th }}$ rib site (port site). Histopathological examination of the mass revealed Actinomycosis. The postoperative period was uneventful and the patient recovered completely. The patient received antibiotic course for 2 weeks. Patient presented 5 months later with swelling in right lumbar evaluated showed fungal hyphae (Eumycetoma) and course completed.
\end{abstract}

Keywords: Actinomycosis, Abdomen, Eumycetoma

\section{Introduction}

Actinomycosis is a rare, chronic debilitating disease and it is difficult to diagnose and treat. Actinomycosis can involve almost any organ system but Actinomycosis of the anterior abdominal wall is rare and follows recent abdominal organ surgery. We describe herein a rare primary anterior abdominal wall Actinomycosis without abdominal organ involvement. Actinomycosis is a sub acute/chronic bacterial infection that affects different body regions. It is caused by gram-positive filamentous anaerobic to microaerophilic bacteria. Actinomyocytes are common saprophytes in the mouth, gastrointestinal tract, and vagina. It may present as chronic suppurative inflammation or sinus formation. Abdominal involvement is usually associated with right-sided infection following appendicitis that causes breakdown of the mucosa.
Isolatedinvolvement of the abdominal wall is extremely rare. Only eight cases have been reported in the English literature. Herein, we report a recently-diagnosedcase of abdominal wall Actinomycosis. Eumycetoma is an uncommon infection, especially as a co-infection with actinomycosis in a same lesion in an adult. Eumycetoma is a chronic cutaneous and subcutaneous infection caused by various genera of fungi, leading to progressive destruction of soft tissue and the nearby anatomical structures. Eumycetoma is mainly a disease of the tropical and subtropical zones, seen in adults, belonging to low socioeconomic status or rural background who are manual workers like agriculturalist and who walk with barefoot in dry, dusty environment. It is characterized by a triad of tumefaction, draining sinuses and presence of colonial grains in the exudates.

\section{Case Report}

A 58-year old male presented to the surgery out-patient department with complains of abdominal pain - right sided and swelling for the past 10 days. Swelling was insidious in onset, gradual in progression to reach the present size. History of occasional fever was present.

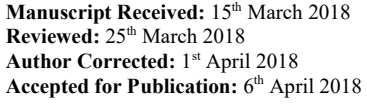




\section{Caes Report}

Patient denied any history of trauma, accidental pricks or injection at that site. Patient had underwentlaparoscopic cholecystectomy 5yrs back and post-operative period was uneventful. Patient is a known case of Type 2 Diabetes Mellitus on Oral Hypoglycemic Agents for 5 months. On admission patient had normal temperature with PR 90/min and normal blood pressure. Abdomen was soft. Palpable mass was present occupying right hypochondrium and right lumbar region. Mass was firm in consistency and was continuous with chest wall. Localised warmth and tenderness were present.

Blood investigations revealed Hemoglobin 9.4g/dl, Total Leucocyte Count 10,200 cells and serum glucose of $225 \mathrm{mg} / \mathrm{dl}$. Chest and abdomen x-ray were normal other than soft tissue shadow in lateral abdominal wall. USG was done which showed an ill-defined collection measuring $10 \mathrm{~cm}$ in diameter in the right hypochondrium close to anterior abdominal wall.

CT abdomen was done with oral and intravenous contrast showed ill-defined peripheral thick walled non-enhancing collection $(13.8 * 4.4 * 12 \mathrm{~cm}$, volume $365 \mathrm{cc})$ seen between the lateral abdominal wall and capsular surface of liver. collection extends to right lateral wall at level of 11 th rib collection is seen causing mass effect on right lobar surface and abutting right latero-conal and posterior renal fascia, no extension into perinephric space. Adjacent sites were normal.
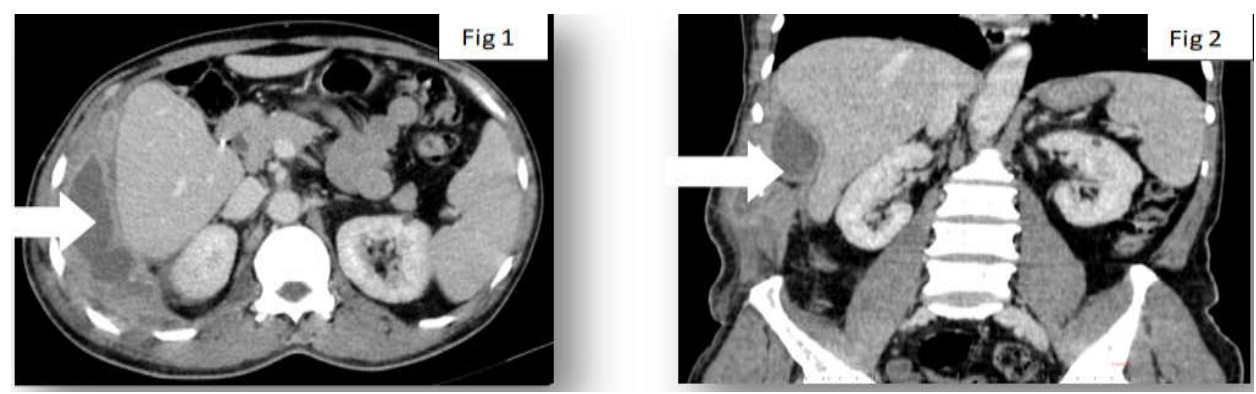

Figure 1 and 2: Computer tomography showing about $13.8 * 4.4 * 1.2 \mathrm{~cm}$ mass projecting from right lower lateral chest wall associated with extension of the right abdominal wall (arrow)

Patient was planned for laparotomy and proceed under GA. intra operative findings - dense adhesions were seen between right lobe of liver and right chest wall. Adhesions were released and no collection was noticed in the sub diaphragmatic space. The chest wall adjoining the liver surface was found to be discolored and sloughed out at places. On poling anabscess cavity was found in the chest wall in the regions of the 10,11 and 12th ribs.

Around $300 \mathrm{ml}$ pus was drained with granules. Biopsy from the wall was taken. Thorough wash was given. Drain tube was placed. Abdomen was closed. Pus was sent for culture sensitivity and fungal culture.

Patient post-operative period was uneventful. HPE showed Actinomycosis. Patient recovered completely and patient was given a course of parenteral tetracycline for 4 weeks.
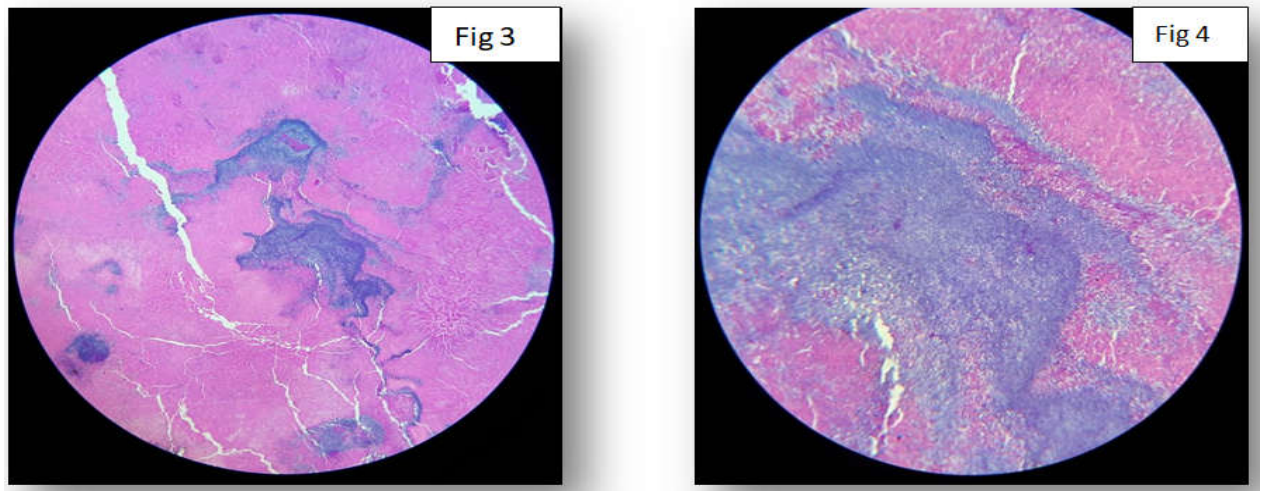

Fig 3 and 4: Showing HPE images of Actinomycosis Low field and High field images 


\section{Caes Report}

Two months after the initial surgery, patient presented with a similar swelling in the same location of size $5 * 3 * 2 \mathrm{~cm}$. USG guided aspiration of contents was attempted but could not be done. Patient was given a course of Inj. amikacin + Tab. cotrimoxazole for a period of 2 weeks. The swelling showed sign of improvement and patient was kept on follow up. The swelling subsided in size with residua induration.

Five months after the initial surgery, the patient presented back to us with a swelling in the right lumbar region. The swelling was fluctuant and was $2 \mathrm{~cm}$ inferior from the right chest wall. Patient was planned for I \& D. Pus was sent for bacterial (aerobic and anaerobic) culture and sensitivity and fungal sensitivity. Culture and sensitivity study revealed presence of fungal hyphae. Patient was started on T. Itraconazole for 2 months. Swelling decreased in size. Patient is on follow up for a period of month after completion of fungal therapy and is asymptomatic.

\section{Discussion}

Actinomycosis is a rare chronic, suppurative pseudo tumoral disease caused by an anaerobic gram positive organism that is most frequently Actinomyces Israeli [1]. In healthy subjects, Actinomyces is a part of normal flora of the oral cavity, the gastrointestinal tract and the genital tract. Healthy mucosa acts as a barrier to the spread of the organism, but tissues damaged by neoplasm, surgery, trauma, or foreign body allow multiplication and spread of Actinomycosis [1].

Actinomycosis most commonly affects the head and neck (50\%) but can involve almost any organ system [1]. Abdominopelvic Actinomycosis is uncommon and typically follows recent bowel surgery such as procedures for acute appendicitis, diverticulitis or abdominal trauma [1].

Primary Actinomycosis of the anterior abdominal wall is very rare and has been reported only in 29 cases in the literature by 2010 [2]. Immunocompromised status, such as diabetes mellitus, steroid therapy, and neoplasm, is a significant predisposing factor for Actinomycosis [3].

Patients usually present with a slowly developing indolent swelling of the soft tissue. The disease usually presents as a slowly growing mass with eventual formation of sinuses discharging serosanguinous pus containing "sulfur" granules, which represent macro colonies of actinomyocytes [3]. The "sulfur" granules are pathognomonic, but these are not always present [3].

In most cases, clinical and diagnostic exams are not able to identify the disease and therefore the treatment becomes complex. Hematological laboratory exams show elevated nonspecific inflammatory markers and serology is not diagnostic [4].

Ultrasonography, computed tomography or MRI does not suffice to differentiate between Actinomycosis and other inflammatory or neoplastic processes [1].
It is also very difficult to isolate the bacterium from cultures [4]. Negative culture rate was reported to be $76 \%$ [3]. The most difficult task for the management of Actinomycosis is to reach a diagnosis before a surgical approach. Preoperative diagnosis is difficult because of non-specific clinical, laboratory and imaging findings. In many cases, the diagnosis is often possible during surgical exploration or after extensive debulking [4].

This is the reason why a wide primary resection including the surrounding tissue is inevitable [1]. Operative management is required for diagnostic biopsy or total excision. Surgery is necessary in many cases, especially when the mass is large or there are some complications such as peritonitis, compression of abdominal organ [4]. Surgery is also necessary when there is no benefit and general health conditions deteriorate after a week of antibiotic treatment [4].

The treatment of choice is proper surgical drainage at the primary site of infection. If a soft tissue tumor is suspected, a wide primary resection including the surrounding tissue is sometimes inevitable. The abdominal wall defect may be closed by prosthetic mesh if the abdominal wall defect is large and direct closure is impossible [1]. Surgical treatment without antibiotic therapy is not always sufficient to achieve a cure for Actinomycosis.

When antibiotic therapy is combined with surgery, it is relatively simple to treat and the cure rate is more than $90 \%$ [5]. Intravenous penicillin is the initial therapy of choice. In the case of penicillin allergy, tetracycline, erythromycin, clindamycin or minocycline are acceptable alternatives [6].

Our patient had positive penicillin skin test so, we started minocycline. The patient will take minocyline for six months. Eumycetoma is mainly a disease of the tropical and subtropical zones, especially between the tropic of cancer and the tropic of Capricorn, that is between latitudes $15 * \mathrm{~S}$ and $30 * \mathrm{~N}$. 


\section{Caes Report}

The disease is commonly seen in adult men. It usually presents between 20 and 50 years of age with male to female ratio 3.5: 1. It is commonly seen in people who live in rural areas in endemic region. Agents that cause Eumycetoma are Primarily Saprophytic microorganisms that are found in the soil and on plant matter. Healthy persons become inoculated with these agents as a result of the traumatic implantation of thorns, Splinters and other Plant matter. Therefore, the incidence of mycetoma is more particularly occupation dependent as people like farmers, herdsmen and fieldworkers are more likely to come in contact with the causative agents.

The most common site is foot as $70 \%$ of all mycetomas affect the foot hence the name madura foot. However, extra pedal involvement also occurs and has been detected in hand, leg, head and neck, abdominal wall, buttock and perineum.

The rural area to which the patient belongs has similar environmental condition, i.e. dry and dusty with thorny vegetation all around. We also postulate that the Tuberculosis was most likely acquired by contact with an infected individual in the community as tuberculosis is endemic in southern tropical region, due to the lowSocioeconomic status ofthe people, malnutrition, low immunity, which predisposes the population to this overwhelming disease.Eumycetoma may present as a small localized tumor likemass, with or without sinuses, or can be associated with significant morbidity in terms of gradual enlargement, destruction and deformity of the affected site.

The diagnosis of Eumycetoma is made tentatively clinically, when discharging grains are visible to the naked eye. The grains vary in colour, size and consistency depending on the causative agent and can be confirmed by culture method. Granules of eumycetoma are firm 0.2 - $5 \mathrm{~mm}$ aggregates of organized, vegetative septate hyphae, which often are embedded in a matrix cement substance of the eumycetoma, producing black granules. M. Mycetomatis accounts for most cases worldwide. Pseudoallescheria boydii is the common aetiologic agent in the United States, while madurellagrisea is a common aetiologic agent in south America.

In general, the geographical distribution of the various mycetoma agents is related to the amount of rainfall and other climatic conditions, and thus, each geographical region has a different list of most common agents. The smears from eumycetoma lesions have a distinct cytological appearance, characterized by brown to black colonies of branching, septate hyphae embedded in a matrix which stain positively with PAS or Gomori's methenamine silver stains, both demonstrating large sized hyphae of eumycetoma.

A diagnosis of eumycetoma should be suspected in case of discharging sinuses, especially those exhibiting black granules. The cytological diagnosis of eumycetoma can be as accurate as histological diagnosis, and techniques such FNAC as well as imprint smears can definitely be taken in to consideration before planning any medical or surgical treatment as these are simple, inexpensive and fairly reliable techniques without any obvious disadvantages. Special fungal stains can also be well applied to cytological specimens for further confirmation, whereas culture studies are helpful in confirmation of diagnosis and species identification.

In literature, we found no reports of Eumycetoma patients coinfected with Actinomycosis in a same lesion [8]. The basis of management includes: clinical examination, high index of suspicion, histopathology, isolation and culture of organism. Eumycetoma are only partially responsive to anti-fungal therapy but can be treated by surgery due to their normally well circumscribed nature. Surgery in combination with azole treatment is the recommended regime. This patient has been on a regular follow-up for 4 months and has shown complete healing of the wound.

\section{Conclusion}

In summary, isolated Actinomycosis of the abdominal wall is an extremely rare clinical entity. The exact pathogenesis remains to be elucidated. Clinicians should be aware of such presentation so that when it is suspected appropriate anaerobic cultures can be taken and tissues carefully examined.

Conflict of interest: None declared. Funding: Nil, Permission from IRB: Yes

\section{References}

1. Ladurner R, Bogner JR, Drosse I, Volkmer E, Sommerey S, Hohenbleicher F, Wirth S, et al. A rare case of primary actinomycosis of the anterior abdominal wall: diagnosis and treatment. Hernia. 2008;12(5):549552.

2. Gomez-Ramirez J, Martin-Perez E, Alcaide B, Martin-Alvarez JL, Larranaga E. Primary abdominal wall actinomycosis. Cir Esp. 2009;85(4):256-258. 


\section{Caes Report}

3. Hefny AF, Joshi S, Saadeldin YA, Fadlalla H, AbuZidan FM. Primary anterior abdominal wall actinomycosis. Singapore Med J. 2006;47(5):419-421.

4. Acquaro P, Tagliabue F, Confalonieri G, Faccioli P, Costa M. Abdominal wall actinomycosis simulating a malignant neoplasm: Case report and review of the literature. World J Gastrointest Surg. 2010;2(7):247250.

5. Das N, Lee J, Madden M, Elliot CS, Bateson P, Gilliland R. A rare case of abdominal actinomycosis presenting as an inflammatory pseudotumour. Int $\mathrm{J}$ Colorectal Dis. 2006;21(5):483-484.
6. Kawahara M, Kawahara K, Goto T, Yamamoto S, Fuchinoue S, Matsumoto T. Abdominal actinomycosis misdiagnosed as a secondary bladder tumor: a case report. Int J Urol. 1998;5(5):498-500.

7. SeockYeolLeea, c, Ji HyeLeeb, Seung JinLeea, Cheol SaeLeea Primary Abdominal Wall Actinomycosis Journal of Medical Cases, ISSN 1923-4155 print, 19234163 online.

8. Rajesh Subramaniam, Shruthikamal Venkat, Reshma Sattar Coexistence of eumycetoma and tuberculosis in a same lesion in right thigh in an adult: a rare case report pISSN 2349-3305 | eISSN 2349-2902.

\section{How to cite this article?}

Agil. S., Ratna, N. Bharath, A. Rekha, A case of primary actinomycosis and secondary eumycetoma in anterior abdomen wall - A case report. Surgical Update: Int J surg Orthopedics.2018;4(4):183-187.doi:10.17511/ijoso.2018.i04.09. 